\title{
The Polarization of the Crab Pulsar with HST
}

Joseph F. Dolan, Patricia T. Boyd ${ }^{*}$ and Robert J. Hill ${ }^{+}$

Laboratory for Astronomy and Solar Physics, NASA Goddard Space

Flight Center, Greenbelt, MD 20771

* also Universities Space Research Association

+ also Hughes STX Corporation

F. Graham-Smith and A. G. Lyne

University of Manchester, Nuffield Radio Astronomy Laboratories, Jodrell Bank, Macclesfield, Cheshire SK119DL, UK

James D. Biggs

Perth Observatory, Walnut Road, Bickley 6076, Western Australia

J. W. Percival

Department of Astronomy and Space Astronomy Laboratory, University of Wisconsin, Madison, WI 53706

Edward L. Robinson

Department of Astronomy and McDonald Observatory, University of Texas, Austin, TX 78712

G. Wayne van Citters

Division of Astronomical Sciences, National Science Foundation, 4201 Wilson Blvd., Arlington, VA 22230

The linear polarization of the Crab pulsar as a function of pulse phase was observed by the High Speed Photometer on the Hubble Space Telescope in March, 1993. Observations were obtained in a bandpass centered on 2770 A using a $0.25 \mathrm{~ms}$ sample time, corresponding to a time resolution of 0.0075 in pulse phase. The UV polarization of the pulsar [Fig. 1] is strikingly similar to that observed in the visible (cf. Smith et al. 1988). The same values of polarization and the same swing of position angle occur through the main and secondary pulses. The polarization pulse profile must be essentially wavelength independent at frequencies above the infrared.

Any model of the emission regions in the Crab pulsar must then be wavelength independent. Our observations support the geometrical model proposed by Smith et al. (1988), which ascribes the double pulse to radiation from regions above the two magnetic poles, and places them at a radial distance of about nine-tenths of the velocity-of-light radius. 


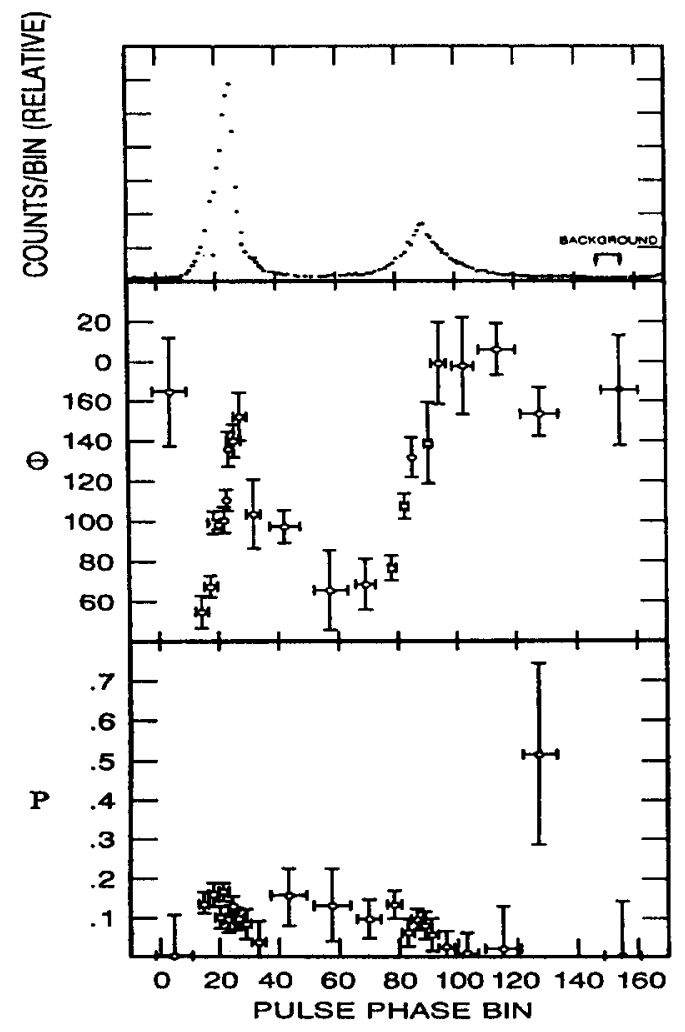

Fig. 1. The polarization of the Crab pulsar in the ultraviolet. One pulse period is divided into 160 equally spaced phase bins at the barycenter of the Solar System. The phase of maximum intensity was arbitrarily assigned to bin 24. Top: the relative intensity as a function of pulse phase in the F160LP bandpass (Percival et al. 1993). The background was taken to be the counting rate in the phase interval denoted. Middle: the position angle of the polarization in the F277M bandpass. Vertical bars denote one standard deviation uncertainties; horizontal bars demarcate the extent of each measurement in pulse phase. Bottom: the fractional polarization, corrected for its non-normal distribution at low statistical significance (Wardle \& Kronberg 1974). The error bars have the same meaning as in the middle panel.

\section{References}

Percival, J. W. et al., 1993, ApJ, 407, 276.

Smith, F. G., Jones, D. H. P., Dick, J. S. B. \& Pike, C. D., 1988 MNRAS, 233, 305.

Wardle, J. F. C. \& Kronberg, P. P. 1974, ApJ, 255, L1. 